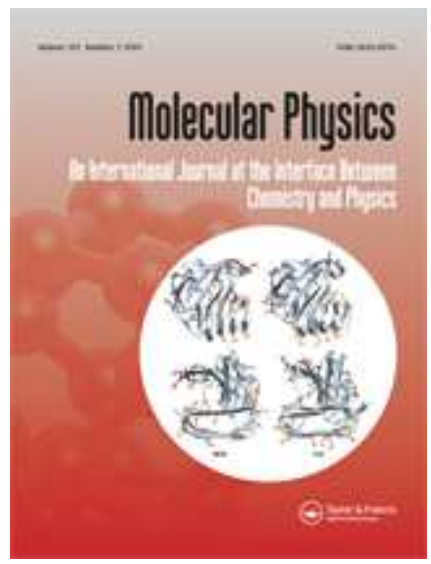

\title{
Accessible volume in quenched-annealed mixtures of hard spheres: a geometric decomposition
}

\begin{tabular}{|c|c|}
\hline Journal: & Molecular Physics \\
\hline Manuscript ID: & TMPH-2010-0480.R1 \\
\hline Manuscript Type: & Special Issue paper - In honour of Bob Evans \\
\hline $\begin{array}{r}\text { Date Submitted by the } \\
\text { Author: }\end{array}$ & 13-Jan-2011 \\
\hline Complete List of Authors: & $\begin{array}{l}\text { Kurzidim, Jan; Technische Universität Wien, Institut für } \\
\text { Theoretische Physik and Center for Computational Materials Science } \\
\text { Kahl, Gerhard; Technische Universität Wien, Institut für } \\
\text { Theoretische Physik and Center for Computational Materials Science }\end{array}$ \\
\hline Keywords: & computational geometry, colloids, disordered confinement \\
\hline \multicolumn{2}{|c|}{$\begin{array}{l}\text { Note: The following files were submitted by the author for peer review, but cannot be converted } \\
\text { to PDF. You must view these files (e.g. movies) online. }\end{array}$} \\
\hline molphys.tgz & \\
\hline
\end{tabular}

\section{SCHOLARONE Manuscripts}


Molecular Physics

Vol. 00, No. 00, Month 200x, 1-16

\title{
SPECIAL ISSUE PAPER
}

\section{Accessible volume in quenched-annealed mixtures of hard spheres: a geometric decomposition}

\author{
Jan Kurzidim* and Gerhard Kahl \\ Institut für Theoretische Physik and Center for Computational Materials Science, \\ Technische Universität Wien, Wien, Austria \\ ( "received")
}

\begin{abstract}
Model systems in which fluid particles move in a disordered matrix of immobile obstacles have been found to be a reasonable representation of a colloidal fluid confined in a disordered porous medium. For systems consisting of hard-sphere particles, the obstacle matrix partitions the space available to the fluid particles into voids of finite volume ("traps") and a percolating void that extends over the entire volume. This geometric distinction plays a key role for the dynamic properties of the confined fluid: while its particles are not able to escape from traps, in the percolating void they can propagate infinitely far. We present a geometric method, based on a Delaunay decomposition, to identify the two different kinds of voids in an arbitrary matrix configuration of finite size under periodic boundary conditions. We subsequently apply a rastering technique, which enables us to statistically characterise the structure of the voids. We investigate the specific case of a quenched-annealed mixture of identical hard spheres, for which, among others, we accurately determine the matrix packing fraction at which the percolation transition of the voids takes place.
\end{abstract}

Keywords: computational geometry, colloids, disordered confinement

\section{Introduction}

In recent years, colloidal fluids confined in porous materials have received considerable attention. Experimental investigations on dozens of different systems evidence that under such conditions a fluid can drastically change its structural, thermodynamic, and dynamic properties. For an overview over the field, we recommend for instance [1-6] and the references therein. A particularly interesting challenge reported in these works, which is yet to be solved, is the dynamic behaviour of fluids that are supercooled in the presence of disordered confinement: in some experimental setups the confined fluid has been shown to be decelerated with respect its bulk counterpart, in other systems an acceleration was observed, and in yet other systems glass transitions were found to vanish [3-6]. Realising that fluids moving in disordered materials play a pivotal role in a broad variety of applied problems ranging from biology over chemistry to geology and technology, it seems essential to pinpoint the microscopic and collective mechanisms responsible for such seemingly contradictory effects.

Unfortunately, a satisfactory theoretical treatment of fluids in disordered confinement has turned out to be difficult, with the major complication being the statistical nature of the confinement. To date, one of the most successful approaches to the problem is the so-called "quenched-annealed" (QA) formalism. In this model, the confinement is represented by the particles of a one-component fluid frozen in place

*Corresponding author. Email: kurzidim@cmt.tuwien.ac.at 
("quenched"); in this array of obstacles the particles of another fluid are allowed to move ("annealed"). Based on initial work by Madden and Glandt [7, 8], Given and Stell $[9,10]$ succeeded in deriving a theoretical framework for QA systems which offers the distinct advantage of using the well-established formalism of statistical physics to account for both the fluid and the confinement. Subsequent works used both theoretical extensions and computer simulations to study in detail the static [11-14] and thermodynamic [14-19] properties of QA systems.

Dynamic properties, however, remained beyond the capabilities and capacities of both theory and simulations for a long time. In the realm of theory, only recently a breakthrough was achieved by Krakoviack: based on similar concepts as those used by Given and Stell, he succeeded in extending the mode-coupling theory of the glass transition (MCT) $[20,21]$ to systems with quenched disorder [22-24]. Probing the applicability of his theory, Krakoviack considered a QA system consisting of a hard-sphere fluid moving in a quenched configuration of another hard-sphere fluid. For this particular system, the theory predicted a number of unusual dynamic features: (1) bulk-like discontinuous glass transitions at low obstacle densities, (2) continuous glass transitions at large obstacle densities, (3) a localisation transition, and (4) a re-entrant scenario at large obstacle densities and low fluid densities.

In recent computer simulation work [25-27] it was found that many of the observed phenomena are captured qualitatively, in some cases even semiquantitatively by the above predictions. However, since MCT is essentially a meanfield theory, it is inherently incapable of making statements concerning the microscopic origin of the observed phenomena. In the specific case of a monodisperse hard-sphere fluid moving in an array of hard-sphere obstacles, this means that MCT is ignorant about the fact that the fluid particles can be classified in two categories. The classification is based on the following consideration: the fixed matrix of hard spheres partitions the system volume in spaces that are accessible to the centre of a fluid particle ("voids") and spaces that are not. The observation fundamental to the present work is that there exist two types of voids: disconnected voids of finite size, and possibly a "percolating" void. The disconnected voids are entirely bounded by infinite potential walls so that a fluid particle placed within cannot escape; such voids will be termed "traps" and fluid particles therein as "trapped" particles. In the percolating void, on the other hand, particles can move infinitely far from their initial locations; such particles will be denoted as "free" particles. (Note that multiple percolating voids can exist only in case of a finite system volume and periodic boundary conditions.)

The QA protocol was specifically devised to describe a homogeneous and isotropic fluid. Therefore, the protocol requires that the usual thermodynamic average (which in a bulk fluid suffices to guarantee isotropy and homogeneity) be complemented with another average which is to be taken over different matrix realisations [10]. Moreover, in order to guarantee that isotropy and homogeneity are fully taken into account, it is imperative to populate both the traps and the percolating void (if present) with fluid particles. However, it is obvious that trapped and free particles differ in many properties, especially dynamic ones, which calls for a study in which these two classes of particles are distinguished. Answering this call, in this work we introduce a method to identify trapped and free particles. Since (as discussed shortly) this method is based on a geometric analysis of the quenched matrix, it yields as a side product a wealth of information about the structure of the confining matrix. These findings will be presented after the core part of this work, the description of separation procedure. Findings obtained from applying the procedure to the fluid component of QA systems have been reported in a separate work [28]. 
Our approach to tackle this problem is based on a Delaunay decomposition that maps the void structure onto a discrete network of sites and bonds [29-32]. This allows not only for the desired identification of traps and percolating void(s), but to also investigate the properties of the matrix by applying the methodology commonly used to describe percolation transitions. Since previous works on QA systems have been concerned with dynamic arrest [25-27], the simulations therein had to extend over long simulation times and considered relatively small ensembles $\left[O\left(10^{3}\right)\right.$ particles]. In such systems, voids extend over the periodic boundaries; since this applies in particular to the possibly-present percolating void, particular care has to be taken when identifying the latter. In this work we present an efficient algorithm to solve this task. Finally, we apply a volume rastering technique to determine the amount of accessible volume of each void (similar to that performed in [32]); this allows to evaluate the void size distribution, and provides a quasi-exact means to determine - given a specific obstacle density - the average fraction of fluid particles that reside in traps. Knowing the latter quantity is essential when combining observables (such as dynamic correlation functions) pertaining to the trapped and the free particles into observables of the full fluid.

The paper is organised as follows: In Sec. 2 we describe the geometric method employed to identify traps, the algorithm to identify percolating voids in finite-size systems, and the rastering technique used to determine the volumes of voids. In Sec. 3 we present the results that these methods yielded for the confining matrix of QA systems. In Sec.4 we discuss the implications of the results, indicating in particular their application to the fluid component of a QA system, and close with concluding remarks.

\section{Model and Methods}

\subsection{Generating the porous matrix}

The positions of the obstacles in a quenched-annealed system are obtained by taking a snapshot of an equilibrated one-component fluid at an arbitrary instant of time. In the specific case of monodisperse hard spheres, the statistics of such a onecomponent fluid is entirely determined by its packing fraction $\phi_{\mathrm{m}}$ (for simplicity, the index "m" for "matrix" used in [25, 27] is retained). Therefore, in this case the parameter space to consider is one-dimensional. We used the Monte Carlo algorithm described in Appendix A of [27] to obtain initial configurations of hard spheres at a prescribed $\phi_{\mathrm{m}}$; subsequently an event-driven molecular dynamics algorithm [33, 34] was used to equilibrate those configurations until the system's mean-squared displacement exceeded $(10 \sigma)^{2}$. Here, $\sigma$ is the diameter of the particles, which serves as the length scale in this work. For the purpose of this investigation, at every value of $\phi_{\mathrm{m}}$ we averaged all quantities of interest over at least 100 independent configurations, irrespective of $N_{\mathrm{m}}$, the number of particles in the obstacle matrix. In all computations, periodic (toroidal) boundary conditions and the nearest-image convention were employed.

\subsection{Delaunay decomposition}

For all matrix configurations obtained this way, we identified for each of the voids therein whether it is disconnected (a "trap") or percolates through the entire system (taking into account the periodic boundary conditions). As mentioned before, the notion of voids refers to the spaces accessible to the centres of fluid particles. The basis of the separation method is constituted by a Delaunay decomposition 
Figure 1. Two-dimensional schematic representation of the steps of the Delaunay decomposition procedure. Detailed explanation: see text. Dark (blue) solid disks: matrix particles. Dark (blue) thick dots: centres of matrix particles. Open circles: Delaunay circles. Medium-shade (pink) filled circles: circles at the centres of which a fluid particle can be placed. Dark (grey) solid circles: circles a the centres of which a fluid particle can not be placed. Medium-shade (pink) triangles: accessible Delaunay triangles. Dark (grey) triangles: inaccessible Delaunay triangles. Light (orange) disks: area at which no fluid particle can be placed. Light thin (green) lines: connecting Delaunay edges. Medium-shade thick (red) lines: non-connecting Delaunay edges. Black line segments: highlighters for the connecting part of a Delaunay edge.

of the simulation volume, in which the centres of the obstacle particles serve as vertices. As has been shown by Sastry and coworkers [30], every Delaunay simplex belongs to no more than one void; hence, knowing the "connectivity" (to be defined later) of the simplices, it is possible to identify disconnected and percolating voids. As has been proven by Kerstein [29], this procedure maps the collection of voids onto a discrete off-lattice network of sites (which may or may not be occupied) and bonds (which may or may not connect).

Fig. 1 shows a two-dimensional schematic drawing of the steps that constitute the Delaunay decomposition procedure. For simplicity, in the following description we will refer specifically to two dimensions; the generalisation to arbitrary dimension $d$ (and specifically to $d=3$ ) as well as a more detailed discussion will be given afterwards. Panel (a) of Fig. 1 depicts a matrix configuration of six hard-disk particles of diameter $\sigma$ that form a void. In panel (b), the centres of these disks are represented by full dots; at those dots a number of circles intersect. Each of these circles exhibits a non-trivial property: exactly three of the particle centres lie on its perimeter, and none inside. There exists a unique set of such "Delaunay circles", which follows from Theorems 5.8 and 5.11 in [35] in conjunction with the fact that the collection of vertices in the dual Voronoi diagram is unique [35]. Panel (c) shows circles that are concentric to the Delaunay circles but reduced in diameter so that they touch the matrix particles. Consider now the fluid particles that move in the array of obstacles: suppose their diameter is $\sigma$, like that of the matrix particles. For any obstacle-touching circle in panel (c) this means that if its diameter is less than $\sigma$, then no particle can be placed at the centre of this circle. Such circles are marked in darker colour in panel (c). Consider now that, as depicted in panel (d), triangles are formed by the matrix particles on the perimeter of each Delaunay circle; these are called "Delaunay triangles". If in panel (c) a fluid particle cannot be placed at the centre of some Delaunay circle, then the entire area of the corresponding Delaunay triangle is inaccessible; hence, those triangles need not be considered in the further steps of the decomposition. In panel (e), superimposed to drawing 
(d), in light colour the area is shown in which no fluid particle of diameter $\sigma$ can be placed. This way, it is verified that no particle can reside in the Delaunay triangles that were previously identified inaccessible. Moreover, the void can now be identified visually, and it is clear that it is a trap since "there is no path to the outside". This intuitive conclusion can be formalised by considering for each edge of a Delaunay triangle just the two particles that define it: if they permit to place a fluid particle at the centre of such a "Delaunay edge", then the two neighbouring triangles are connected. The result of applying this criterion is depicted in panel (f), where the matrix particles are now blanked out. The Delaunay edges are highlighted according to their connectivity: the thick red edges can not be passed by a fluid particle, whereas the thin green edges permit passing (indicated by the black bars). As can be seen in the leftmost Delaunay triangle of panel (e), it is possible that a fluid particle cannot be placed within a triangle although it can be placed at the centre of the corresponding Delaunay circle. This is because the centre of the circle can reside outside the triangle. Nevertheless, some edge of such a Delaunay triangle can formally be connecting; since the other edges cannot be passed, the connectivity of the Delaunay triangles correctly reflects connectivity of the voids even in this case.

In the $d$-dimensional case, and hence the case $d=3$ relevant to this work, the following generalisations apply. (i) The notion of Delaunay circles is replaced by that of Delaunay $d$-spheres (spheres of dimension $d$ ); they are defined by the centres of $(d+1)$ fluid particles ${ }^{1}$ that reside on their surface, and fulfil the requirement that no particle centres are located within them. (ii) The Delaunay $d$-spheres define Delaunay $d$-simplices; in $d=3$ they have tetrahedron topology, i.e., four vertices, six edges, and four triangular faces. For this reason, we will refer to the Delaunay 3 -simplices as "Delaunay tetrahedra"; note, however, that the triangle faces of a Delaunay simplex are generally not equilateral. (iii) The Delaunay $d$-simplices are bounded by surface elements of dimension $(d-1)$ which are defined by $(d-1)$ particles. In $d=3$ they consist of triangles which we will refer to as "Delaunay faces". Such a surface element is connecting if at the centre of its circumhypersphere [defined by the $(d-1)$ particles] a fluid particle can be placed.

\subsection{Percolation algorithm}

After completion of the decomposition procedure, for each Delaunay tetrahedron it is known whether its circumsphere is accessible to the centre of a fluid particle, and for each Delaunay face it is known whether it is connecting or not. In the network picture that was previously alluded to [29], the tetrahedra correspond to sites and the faces represent bonds: both of these can be occupied or not, but bonds can exist only between occupied sites. In the next step, these building blocks are assembled into connected groups, each of which describes a void. In case that a group consists of a finite number of members, this is a relatively minor challenge; if on the other hand the group spans across the simulation box (considering the periodic boundary conditions), it is vital to avoid a multiple counting of tetrahedron images while still being able to identify whether the void is percolating. For this task, we devised a novel algorithm, as described in the following.

To allow for a visual description (see later), we will again employ the terminology of two dimensions; the generalisation of the procedure to three dimensions is

\footnotetext{
${ }^{1}$ Note that there may exist spheres with more than $(d+1)$ particle centres on their surface and none inside; in this case a single sphere defines more than one Delaunay $d$-simplex. Since there is no unique way to define the Delaunay $d$-simplices inside such $d$-spheres, the resulting Delaunay diagram is called "frustrated".
} 


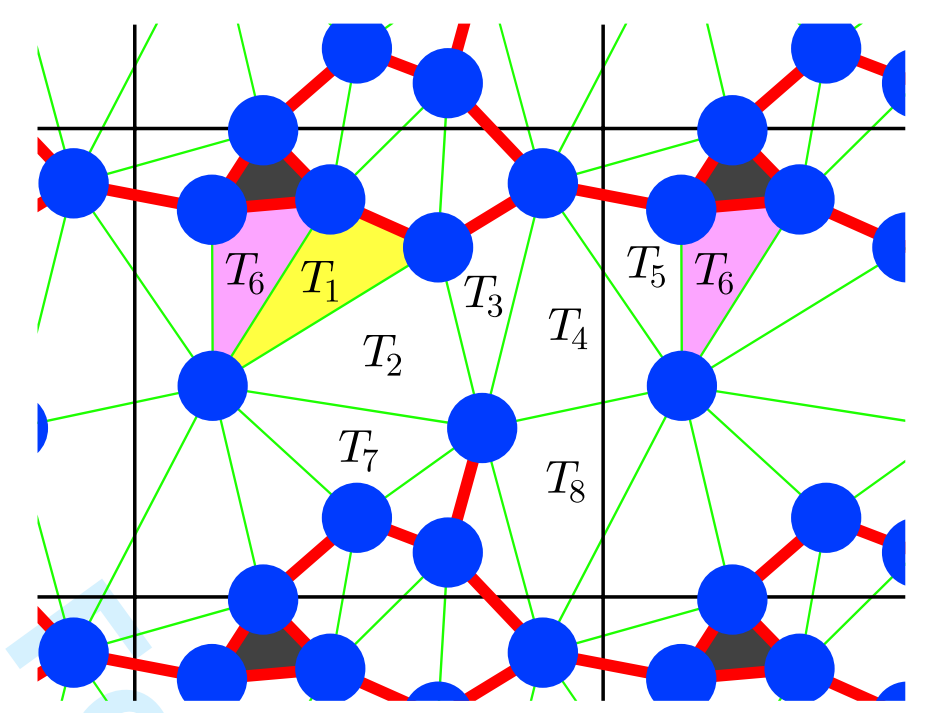

Figure 2. Two-dimensional schematic representation of a complete Delaunay decomposition of a random arrangement of eight obstacles with periodic boundary conditions. Dark (blue) disks: matrix particles. Medium-shade thick (red) lines: non-connecting Delaunay edges (see Fig. 1). Light thin (green) lines: connecting edges. Thin black lines: box borders, separating periodic images. Dark (grey) areas: non-accessible Delaunay triangles. Light (yellow) area: initial triangle in the procedure to determine the connectivity of the triangle groups. Medium-shade (pink) triangle: first triangle for which a periodic image is encountered in that procedure.

straight-forward. The starting point is marked by choosing at random a Delaunay triangle of which it is not yet known to which group of triangles it belongs. First, we add this triangle to a list and store its location (for instance the centre of the corresponding Delaunay circle) as the origin of the triangle group. We then consider each Delaunay triangle that borders the current triangle via a connecting edge: First, we compute its location by adding the difference vector between the bordering and the current triangle to the position of the latter. Then, we check if the bordering triangle is already an element of the list: If not, we add it to the list and store its location. If it is already contained in the list then the stored location of the triangle is retrieved and compared with the location just computed; if those locations do not match then the newly-found triangle is a periodic image of the one already contained in the list, and the triangle group defined by the list must constitute a percolating void. After checking all of the bordering triangles, the current triangle is marked "processed"; then, the unprocessed triangle with the lowest identifier is retrieved from the list, and the same procedure is repeated. This is iterated until the list contains solely processed triangles; the list then contains all triangles in the triangle group.

To clarify this procedure, consider the schematic drawing shown in Fig. 2. Suppose at the beginning the triangle $T_{1}$ is the only element of the list and its location has been stored. Then, in the first loop, $T_{2}$ and $T_{6}$ are added to the list. In the second loop $T_{1}, T_{3}$, and $T_{7}$ are considered, but since $T_{1}$ is encountered at the same location as previously saved, it is discarded and only $T_{3}$ and $T_{7}$ are added to the list. In the third loop, only $T_{4}$ is added; in the fourth loop this is done for $T_{5}$ and $T_{8}$. In the fifth loop, finally, $T_{6}$ is encountered, but at a location differing from the encounter in the first loop. Therefore, the group must be percolating. In order to find the remaining triangles, the procedure is then continued at $T_{6}$, which yields no additional entries to the list, and then at $T_{7}$.

After applying the same dimensionality generalisations as before, the three dimensional procedure is described by the following pseudo-code:

while list contains unprocessed tetrahedra do 
Figure 3. Two-dimensional schematic drawing of the rastering technique applied to each Delaunay simplex to determine the volume therein that is accessible to the centre of a fluid particle. Each square represents one rastering grain. Dark (blue) disks: matrix particles. Light (orange) disks: non-accessible area. Black empty triangle: a Delaunay triangle. Empty squares: grains that are not located within the triangle. Dark (grey) squares: inaccessible grains that belong to the triangle. Medium-shade (pink) squares: accessible grains that belong to the triangle.

\author{
choose unprocessed tetrahedron with lowest id \\ for all tetrahedra bordering a connecting face do \\ compute location \\ if not on list then \\ save location, add to list \\ else if computed location $\neq$ stored location then \\ mark group "percolating" \\ end if \\ end for \\ mark current tetrahedron "processed" \\ end while
}

\title{
2.4. Volume rastering technique
}

The procedure described so far is sufficient to distinguish between traps and the percolating $\operatorname{void}(\mathrm{s})$. However, in order to analyse in more detail the statistics of the voids, it is useful to also determine their volumes, i.e., the amount of space accessible to the centre of a fluid particle in each void. This allows for instance to trace out void size distributions, which may then be used to interpret the dynamic behaviour of fluid particles moving in the obstacle array. Also, knowing the void volumes renders it possible to precisely evaluate the average fraction of trapped particles in QA systems. Moreover, consider that the voids undergo a percolation transition [36] when varying the matrix density $\phi_{\mathrm{m}}$ : above a certain $\phi_{\mathrm{m}}^{*}$ no percolating void exists. In an infinitely-large system, this transition is sharp; however, in systems of finite size more care has to be taken to reliably identify $\phi_{\mathrm{m}}$. In this context knowledge about the void volumes is useful, although it also possible to conduct investigations on this phenomenon based solely on the Delaunay decomposition [31].

Sastry and coworkers [30] developed a complete, but rather complicated formalism to exactly determine the size of a void. Since for the purpose of this work we were merely interested in a reliable estimate of the void sizes, we opted for a simpler approach like done for instance in [32]. In this approach, the volume is rastered by considering a large number of cube-shaped "grains" arranged in a simple cubic lattice: first, for each Delaunay tetrahedron the grain centres located 
inside the tetrahedron are determined; subsequently, for each of the corresponding grains it is checked whether the centre of a fluid particle can access the grain centre. The accessible volume inside a single tetrahedron is then the number of accessible grains times the volume of a grain, the latter being simply the lattice spacing to the third power. In combination with the information about the connectivity of the Delaunay tetrahedra, the grains allows to evaluate the volume of each void. The rastering procedure is visualised in Fig. 3, where accessible grains located inside the tetrahedron are indicated by squares shaded in a medium (pink) tone, whereas grains that belong to the tetrahedron but are not accessible are represented by dark (grey) squares. The lattice depicted in Fig. 3 features merely six grains per particle diameter; this resolution is considerably coarser than the one used in our investigations. For computing most observables we employed a lattice spacing of 15 grains per particle diameter; for determining the unweighted void size distribution (Fig. 7 in Sec. 3.2) we increased this figure to as much as 100. Unless stated otherwise in Sec. 3, uncertainties associated with the finite size of the grains are much less significant than statistical errors. 


\section{Results}

\subsection{Statistics of the Delaunay decomposition}

Before we turn to a detailed discussion of the voids, we investigate some of the statistical features of the underlying Delaunay decomposition and the accessible volume. For this, no information about the notion of traps and percolating voids is required. In Fig. 4, three quantities are presented that vary with the matrix packing fraction, $\phi_{\mathrm{m}}$, and can easily be extracted from the procedure discussed in Sec. 2. Panel (a) shows $P_{\text {tetrhed }}\left(\phi_{\mathrm{m}}\right)$, the probability that a fluid particle can be placed inside the circumsphere of a Delaunay tetrahedron; panel (b) depicts the related quantity $P_{\text {face }}\left(\phi_{\mathrm{m}}\right)$, the probability that a Delaunay face is connecting. Panel (c) presents $P_{\text {grain }}\left(\phi_{\mathrm{m}}\right)$, the probability that a grain centre is accessible to a fluid particle. The latter quantity can of course be calculated without the Delaunay decomposition; it merely represents the overall volume accessible to the centre of a fluid particle. By combining Eq. 2 in [37] and Eq. 2.7 in [38], it is also possible to obtain the analytic expression

$$
P_{\text {grain }}^{\mathrm{CS}}\left(\phi_{\mathrm{m}}\right)=\exp \left\{-\frac{8 \phi_{\mathrm{m}}-9 \phi_{\mathrm{m}}^{2}+3 \phi_{\mathrm{m}}^{3}}{\left(1-\phi_{\mathrm{m}}\right)^{3}}\right\}
$$

for the accessible volume; the derivation is based on the Carnahan-Starling (CS) equation of state [39] and the excess chemical potential. (Note that the index "grain" was retained for notational simplicity only; the derivation is ignorant of the concept of grains.) For comparison, $P_{\text {grain }}^{\mathrm{CS}}$ is included in Fig. 4(c) as a light grey background curve.

Anticipating that the $\phi_{\mathrm{m}}$ range considered in Fig. 4 includes $\phi_{\mathrm{m}}^{*}$, the density at which the percolation transition of the voids takes place (indicated by the dashed vertical line), the most striking feature of these three probabilities is that nothing even remotely indicates this fact. On the contrary, none of the quantities considered decreases by more than a factor of two over the entire range of $\phi_{\mathrm{m}}$, and all do so in a strictly monotonic fashion. Therefore, as has been discovered before [31, 37], a simple analysis of $P_{\text {grain }}$ cannot be capable of predicting the correct dynamics of a QA mixture if $\phi_{\mathrm{m}}$ is close to $\phi_{\mathrm{m}}^{*}$, and neither could an analysis based on the other two probabilities. As expected, $P_{\text {tetrhed }}, P_{\text {face}}$, and $P_{\text {grain }}$ are found to be independent of the size of the system; thanks to the large number of averaged elements, the curves for different system sizes are virtually indistinguishable and errors are minute. The accuracy of the data is even more evident from panel (c), where they are superposed to $P_{\text {grain }}^{\mathrm{CS}}$, which can be regarded to be essentially exact. As an interesting side finding (not shown in a plot), we determined the that average number of Delaunay tetrahedra per matrix particle, $n_{\text {tetrhed }}\left(\phi_{\mathrm{m}}\right)$, monotonically decreases from $n_{\text {tetrhed }}(0.235)=6.584 \pm 0.003$ to $n_{\text {tetrhed }}(0.270)=6.547 \pm 0.003$. Note that in a periodic system the number of Delaunay faces is exactly twice that of the tetrahedra.

\section{2. $\quad$ Statistics of the voids}

Figure 5 gives a visual impression of a Delaunay decomposition (a) and of the subsequent volume rastering (b) for a sample system containing $N_{\mathrm{m}}=714$ matrix particles; the latter number is typical for the simulations performed in [25, 27, 28]. In both panels, elements coded in darker (red) shade constitute traps, whereas elements in lighter (green) shade belong to the percolating void. In panel (a), only Delaunay tetrahedra were considered for which a fluid particle can be placed at 


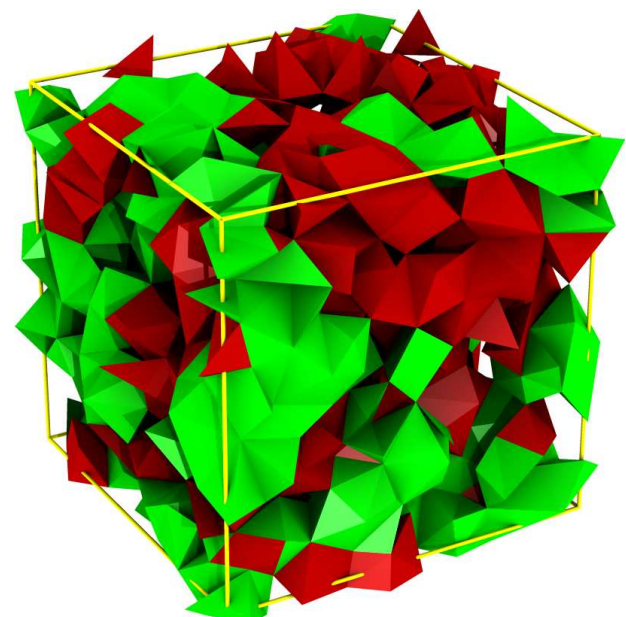

(a)

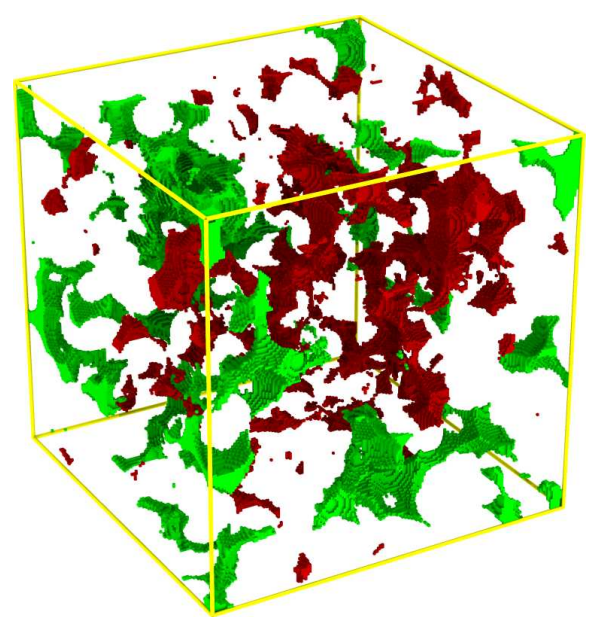

(b)

Figure 5. Three-dimensional representation of the objects resulting from a Delaunay decomposition and a subsequent rastering (cf. Sec. 2) applied to a sample matrix consisting of 714 particles. Dark (red): objects pertaining to traps. Light (green): objects that constitute the percolating void. (a) Delaunay tetrahedra for which a fluid particle can be placed at the circumsphere centre. (b) Grains for which a fluid particle can be placed at the centre.

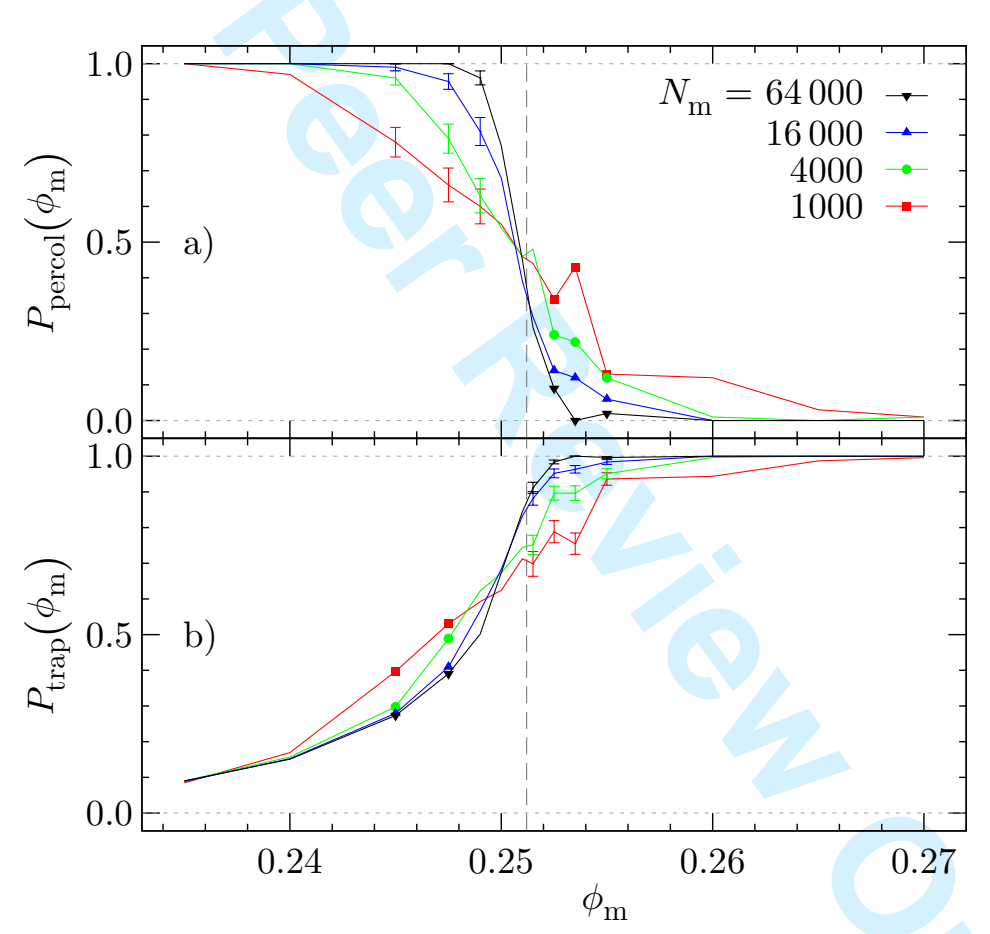

Figure 6. Probabilities indicating the presence of a percolation transition which can be extracted from the percolation analysis and the rastering procedure. (a) Probability that a system at a given $\phi_{\mathrm{m}}$ contains a percolating void. (b) Probability that a given grain belongs to a trap. Error bars and vertical dashed line: see Fig. 4.

the centre of the circumsphere. Panel (b) indicates that for the major part of the accessible volume, the rastering technique yields results of adequate accuracy; merely for very small voids the method suffers from inaccuracies. However, since the latter voids comprise only a minute fraction of the accessible volume, those inaccuracies should have negligible impact when analysing the dynamics of an annealed fluid in conjunction with information about the void sizes. From panel (b), it is also evident that the structure of the accessible volume is highly nonuniform, with the voids covering a large range in size and shape.

The most straight-forward quantity to extract from the percolation analysis de- 
scribed in Sec. 2.3 is of course the probability $P_{\text {percol }}\left(\phi_{\mathrm{m}}\right)$ that a system contains a percolating void; naturally it provides one means to estimate $\phi_{\mathrm{m}}^{*}$, the percolation threshold of the void space. $P_{\text {percol }}$ depends not only upon the matrix packing fraction $\phi_{\mathrm{m}}$, but also on the size of the system, here quantified by the number of matrix particles $N_{\mathrm{m}}$. Since periodic boundary conditions were employed, with respect to an infinitely-large system $P_{\text {percol }}$ in small systems is enhanced above $\phi_{\mathrm{m}}^{*}$, while below $\phi_{\mathrm{m}}^{*}$ the reverse is true. From Fig. 6, panel (a), this smearing-out in small systems is immediately evident; such finite-size effects are well-known from order parameters in first- and second-order phase transitions where they are associated with cutting off a divergent length scale (see, e.g., [40]). As expected, $P_{\text {percol }}$ approaches a step function as $N_{\mathrm{m}}$ is increased. An estimation of $\phi_{\mathrm{m}}^{*}$ is provided by the interval over which $P_{\text {percol }}$ changes from 1 to 0 for the largest system considered. This procedure yields $0.248<\phi_{\mathrm{m}}^{*}<0.254$, an interval that indeed encloses the more precise value that we will later extract from Fig. 8 , indicated by the vertical dashed line in Fig. 6. Note that it is a coincidence that the $P_{\text {percol }}$ curves for different $N_{\mathrm{m}}$ intersect at points distributed over a narrow $\phi_{\mathrm{m}}$ range; this is unrelated to scaling considerations and does not serve as a more precise estimate [36].

Panel (b) of Fig. 6 shows another interesting quantity, namely $P_{\text {trap }}\left(\phi_{\mathrm{m}}\right)$, the fraction of accessible volume that is located in traps (as opposed to the percolating void). As expected, $P_{\text {trap }}$ approaches unity as $\phi_{\mathrm{m}}$ is increased towards $\phi_{\mathrm{m}}^{*}$; finitesize effects similar to those in $P_{\text {percol }}$ can be observed. However, unlike the latter, $P_{\text {trap }}$ does not converge to a step function as $N_{\mathrm{m}}$ is increased since below $\phi_{\mathrm{m}}$ traps are encountered in systems of any size. Consequently, distilling the interval containing $\phi_{\mathrm{m}}^{*}$ from $P_{\text {trap }}$ is somewhat less precise than extracting it from $P_{\text {percol }}$. The significance of $P_{\text {trap }}$ lies in a different reason: recall that for setting up QA systems the particles of the fluid component are inserted into the matrix at random positions $[25,27]$. Considering this, it is clear that $P_{\text {trap }}$ describes precisely the average fraction of fluid particles that are located in a trap. Therefore, $P_{\text {trap }}$ at a certain $\phi_{\mathrm{m}}$ can be used to obtain some observable of the full fluid by performing (at the same $\phi_{\mathrm{m}}$ ) an appropriately-weighted average of the same observable pertaining to the trapped and to the free particles.

As mentioned previously, voids of vastly differing size and shape are present in a QA system. The latter finding is not unexpected and can be quantified in a more succinct fashion by computing, at fixed $\phi_{\mathrm{m}}$, the probability distribution of void volumes. First, recognise that given a system at a specific $\phi_{\mathrm{m}}$ and $N_{\mathrm{m}}$, the volume of the percolating void(s) is known as

$$
V_{\text {percol }}\left(\phi_{\mathrm{m}}\right)=\left[1-P_{\text {trap }}\left(\phi_{\mathrm{m}}\right)\right] P_{\text {grain }}\left(\phi_{\mathrm{m}}\right)\left[\frac{\pi}{6} N_{\mathrm{m}} \phi_{\mathrm{m}}^{-1}\right] .
$$

Since this means that $V_{\text {percol }}\left(\phi_{\mathrm{m}}\right)$ can be calculated solely from quantities already discussed, in the following we will consider only traps.

In Fig. 7(a) we present the probability $P(V)$ that a given void contains an accessible volume $V$; panel (b) shows the same quantity weighted with $V$. In the case of $P(V)$, the probability distribution exhibits interesting features over a large range of void volumes (note the logarithmic volume scale in Fig. 7), including in particular very small values of $V$. In order to trace out the distribution to such small volumes, we considered systems containing merely 100 particles. This necessity arose from the fact that a small grain volume entails a large number of grains, and from the fact that computer memory is limited. However, considering that $P(V)$ does not show interesting features above $V \sim 10^{1}$, the choice of $N_{\mathrm{m}}=100$ is sufficient. Using a grain edge length $L_{\text {grain }}=0.01$ (i.e., 100 grains per particle diameter), the systems comprised $N_{\text {grain }} \sim 600^{3}$ grains; this allowed reliable calculations of void 


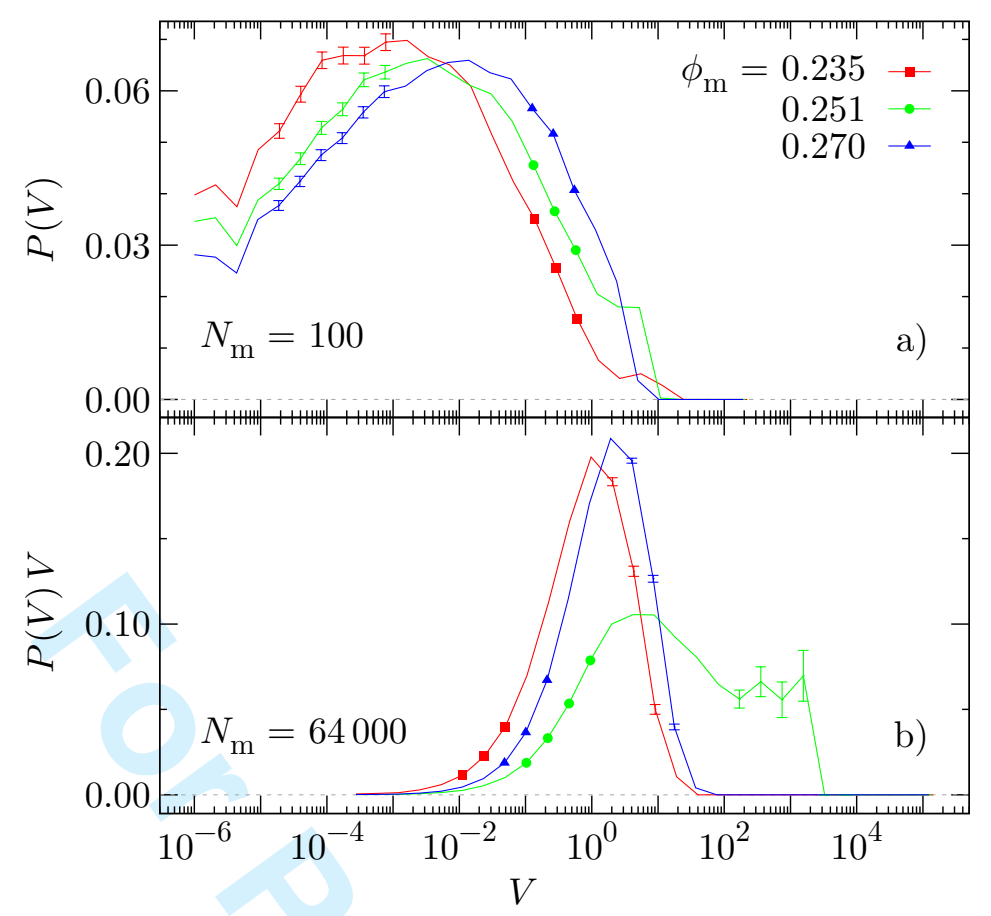

Figure 7. Probability that a given void extends over an accessible volume $V$. Curves represent matrix packing fractions $\phi_{\mathrm{m}}$ below, close to, and above the percolation transition. (a) Unweighted probability, for $N_{\mathrm{m}}=100$ matrix particles. (b) Probability weighted with $V$, for $N_{\mathrm{m}}=64000$. Error bars: see Fig. 4 .

volumes for voids exceeding $V \sim 10^{-5}$. Below this value of $V$, data in Fig. 7(a) suffers from finite-size effects; this concerns in particular the upward "kink" in this range of $V$.

The most interesting feature of Fig. 7 (a) is the fact that there is, almost like in Fig. 4, little (if any) indication of a percolation transition. Merely a small shoulder for $\phi_{\mathrm{m}}=0.251$ and $V \sim 10^{1}$ hints at this fact. Instead, the location of the distribution peak continuously shifts to larger values as $\phi_{\mathrm{m}}$ is increased. Conversely, the volume-weighted distribution $P(V) V$, presented in panel (b), shows clear evidence of a percolation transition. Since by definition this quantifier emphasises large volumes, differences therein are enhanced: For $\phi_{\mathrm{m}}=0.251$, the distribution assumes values of 0.05 for volumes as large as $V \sim 10^{3}$; for the other two densities $P(V) V$ falls off to lower values already at volumes smaller by two orders of magnitude. Considering that $P(V) V$ is essentially zero beyond $V=10^{4}$ and that for $N_{\mathrm{m}}=64000$ and $\phi_{\mathrm{m}}=0.251$ the total system volume is $\sim 50^{3} \sim 10^{5}$, uncertainties in $P(V) V$ arise solely from statistics and not from the finite size of the system.

Finally, we turn to the quantity that-disregarding finite-size scaling techniques - allows for the most accurate [36] determination of the percolation transition: the second moment of the void volume distribution

$$
\langle V\rangle_{\mathrm{vol}}\left(\phi_{\mathrm{m}}\right)=\frac{\int_{0}^{\infty} P(V) V^{2} d V}{\int_{0}^{\infty} P(V) V d V} \simeq \frac{\sum_{i=1}^{N_{\text {voids }}} V_{i}^{2}}{\sum_{i=1}^{N_{\text {voids }}} V_{i}} .
$$

Here, $V_{i}$ denotes the volume of void $i$ in a configuration at a given value of $\phi_{\mathrm{m}}$; note

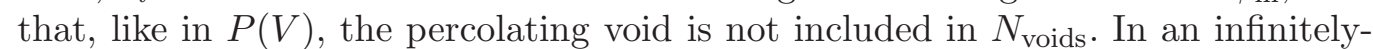
large system the latter quantity diverges at $\phi_{\mathrm{m}}^{*}$; a convergence to this behaviour can immediately be seen from Fig. 8(a). The divergence can easily be rationalised: First, 


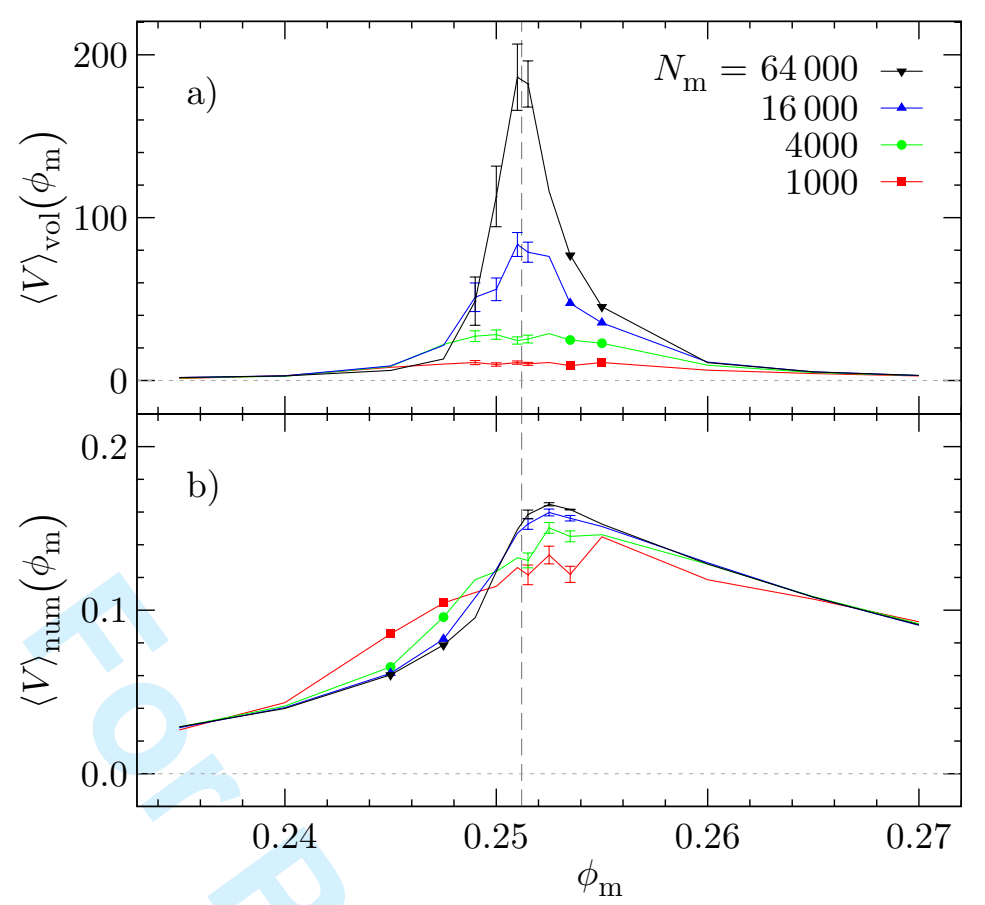

Figure 8. Average volume of the voids formed by the matrix, depending of the matrix packing fraction $\phi_{\mathrm{m}}$. (a) Volume-weighted average $\langle V\rangle_{\text {vol }}\left(\phi_{\mathrm{m}}\right)$. (b) Number-weighted average $\langle V\rangle_{\text {num }}\left(\phi_{\mathrm{m}}\right)$. The vertical dashed line represents the best estimate of the percolation threshold $\phi_{\mathrm{m}}^{*}$ based on position of the peak in panel (a). Error bars: see Fig. 4. [Note that panel (a) of this figure corresponds to panel (b) in Fig. 7, and vice versa].

consider that just above the percolation threshold very large voids exist, which are "almost" percolating in the sense that only in few locations a connection is disrupted by the matrix. Just below the percolation transition voids are also large, since with an increasing number of obstacles substantial accessible volumes are disconnected from the percolating void. Realising, then, that $\langle V\rangle_{\mathrm{vol}}$ is the volumeweighted version of the average void size

$$
\langle V\rangle_{\text {num }}\left(\phi_{\mathrm{m}}\right)=\frac{1}{V} \int_{0}^{\infty} P(V) V d V \simeq \frac{1}{N_{\text {voids }}} \sum_{i=1}^{N_{\text {voids }}} V_{i}
$$

it is clear that a large void will have large weight. Since in we excluded the percolating void, for $\phi_{\mathrm{m}} \neq \phi_{\mathrm{m}}^{*}$ no infinite weight can be present in $\langle V\rangle_{\mathrm{vol}}$. Therefore, the latter can diverge only at $\phi_{\mathrm{m}}^{*}$. For completeness, in panel (b) of Fig. 8 we also show $\langle V\rangle_{\text {num }}$; note, however, that the maximum of the latter does not correspond to $\phi_{\mathrm{m}}^{*}$. Focusing, finally, on the position of the peak in $\langle V\rangle_{\mathrm{vol}}$, the best estimate emanating from the present work for the percolation threshold in QA systems of equal hard spheres is $\phi_{\mathrm{m}}^{*} \simeq 0.251(2 \pm 2)$.

\section{Discussion and Conclusions}

The primary objective of this work was to introduce a geometric method that reliably identifies which of the voids formed by an arbitrary matrix of immobile hard spheres are of finite volume and which are infinitely large. In Sec. 2 we presented an efficient algorithm based on a Delaunay decomposition that suits this requirement specifically in the presence of periodic boundary conditions. Given a set of mobile hard-sphere particles that occupy random locations within such a matrix, this method provides a clear-cut means to determine which among the mobile particles 
are "trapped" (located in a void of finite size) and which are "free" (located in an infinitely large void). It is worth noting that although trapped particles may not be relevant or not even realised in experimental setups, it is of paramount importance to explicitly include these particles when testing theoretical frameworks that explicitly require their presence - only this way such theories, which represent the state of the art to describe fluids in disordered confinement, can be scrutinised and subsequently improved.

The method introduced in Sec. 2 also enables to evaluate various statistical properties of the voids formed by such matrices, which is interesting and useful on its own right. In Sec. 3 of this work, we chose to focus on matrices quenched from an equilibrated hard-sphere fluid since such matrices are the key feature of QA systems. The result that stands out from this study is the highly accurate determination of $\phi_{\mathrm{m}}^{*}$, the packing fraction at which the percolation transition of the voids takes place for QA systems of identical hard spheres. This percolation transition is intimately connected to the dynamic arrest of the fluid particles that move in the host matrix, and its determination using the method introduced in this work provides an independent verification of previous investigations such as Refs. $[25,27,28,41]$. We determined the percolation transition to take place at $\phi_{\mathrm{m}}^{*}=$ 0.2512 , which is in excellent agreement with the findings of the above works, which unanimously found the fluid to exhibit subdiffusive behaviour for $\phi_{\mathrm{m}} \sim 0.25$ (or slightly above this value) and in all cases this fact was attributed to the underlying percolation transition of the voids. There is an apparent slight disagreement with the investigation by Sung and Yethiraj on the "random matrix" protocol [31], for which they found the percolation transition to take place at $\phi_{\mathrm{m}} \sim 0.24$. However, considering that in this protocol the matrix particles are serially inserted into the simulation volume and remain fixed at their insertion locations (see [31] for details), this discrepancy can probably be attributed to the fact that random matrices of this kind do not represent configurations of an equilibrated fluid. This would be in agreement with the finding of the same authors, as well as other authors [26, 32, 42], that $\phi_{\mathrm{m}}^{*}$ depends sensitively on details of the matrix preparation protocol.

There are several possible applications and routes of action emerging from the geometric method presented in this contribution. One of the most straight-forward uses, namely performing a separation in trapped and free particles for QA systems of equal hard spheres, has been been investigated by the authors in a concurrent publication [28]. The results in that contribution have turned out to provide interesting insights into the capabilities of MCT to describe the dynamics of dense QA systems. Furthermore, it is possible to apply our method without modification to systems in which the fluid particles have a radius different from that of the matrix particles, and even to systems with polydisperse fluid particles. In the latter case, the only caveat is an increasingly involved analysis since the void connectivity has to be evaluated separately for each fluid particle, which unfortunately also renders the interpretation intricate. Our method is not directly suited for application to matrices consisting of polydisperse particles; however, it can be extended to this case in a straight-forward façon by making use of the solutions to the so-called "Apollonius' Problem" (i.e., the problem to find a circle that touches three other circles) and its higher-dimensional generalisations in appropriate places of the algorithm. In this context it is due to mention a different route to geometrically analysing polydisperse matrices which has been explored by Sastry and coworkers, who extended the Voronoi construction (which is dual to the Delaunay decomposition) to this case [30]. Another natural application of our method would be to analyse matrices constituted by particles that interact through a continuously varying pair potential. If the hard-sphere potential represents a low-temperature 
limit of such a potential (which is the case, e.g., for the Lennard-Jones potential) the decomposition method could be used to perform a separation in regions of low and high energy, as has been done in Refs. [37, 43].

Finally, also the quantities presented in Sec. 3 and related descriptors of the void structure offer promising routes to directly compute the dynamics of a confined fluid from structural information of the host matrix. For instance, advanced simulational methods can be devised, like the Monte Carlo simulations on Voronoi networks performed in [31], and theoretical approaches can be worked out which involve not only the total accessible volume [37] but also the distributions of the void volumes and of the void connectivities. This way, hopefully, answers can be provided to the dire need for improved theories and extended simulations on fluids in disordered confinement, to thus finally rationalise the existing plethora of experimental information in this field.

\section{Acknowledgements}

The authors are indebted to D. Coslovich for many discussions essential to this work. Valuable comments by J. C. Gimel and A. Yethiraj are gratefully acknowledged. This work was funded by the Austrian Science Foundation (FWF) under Project No. P19890-N16.

\section{References}

[1]L.D. Gelb, K.E. Gubbins, R. Radhakrishnan and M. Sliwinska-Bartkowiak, Rep. Prog. Phys. 62 (12), 1573 (1999).

[2] M.L. Rosinberg, in New Approaches to Problems in Liquid State Theory, edited by Carlo Caccamo, Jean Pierre Hansen and George Stell (Kluwer Academic Publishers, Dordrecht, 1999).

[3] G.B. McKenna, J. Phys. IV France 10 (PR7), 343 (2000).

[4] G.B. McKenna, Eur. Phys. J. E 12 (1), 191 (2003).

[5]M. Alcoutlabi and G.B. McKenna, J. Phys.: Condens. Matter 17 (15), R461 (2005).

[6] G.B. McKenna, Eur. Phys. J. Special Topics 141 (1), 291 (2007).

[7]W.G. Madden and E.D. Glandt, J. Stat. Phys. 51 (3-4), 537 (1988).

[8]W.G. Madden, J. Chem. Phys. 96 (7), 5422 (1992).

[9]J.A. Given and G. Stell, J. Chem. Phys. 97 (6), 4573 (1992).

[10]J.A. Given and G.R. Stell, Physica A 209 (3-4), 495 (1994).

[11]E. Lomba, J.A. Given, G. Stell, J.J. Weis and D. Levesque, Phys. Rev. E 48 (1), 233 (1993).

[12]C. Vega, R.D. Kaminsky and P.A. Monson, J. Chem. Phys. 99 (4), 3003 (1993).

[13] A. Meroni, D. Levesque and J.J. Weis, J. Chem. Phys. 105 (3), 1101 (1996).

[14]E. Paschinger and G. Kahl, Phys. Rev. E 61 (5), 5330 (2000).

[15] M.L. Rosinberg, G. Tarjus and G. Stell, J. Chem. Phys. 100 (7), 5172 (1994).

[16]E. Kierlik, M.L. Rosinberg, G. Tarjus and P. Monson, J. Chem. Phys. 103 (10), 4256 (1995).

[17]E. Kierlik, M.L. Rosinberg, G. Tarjus and P.A. Monson, J. Chem. Phys. 106 (1), 264 (1997).

[18] M. Álvarez, D. Levesque and J.J. Weis, Phys. Rev. E 60 (5), 5495 (1999).

[19]E. Paschinger, D. Levesque, G. Kahl and J.J. Weis, EPL 55 (2), 178 (2001).

[20] W. Gotze and L. Sjogren, Rep. Prog. Phys. 55 (3), 241 (1992).

[21] W. Götze, J. Phys.: Condens. Matter 11 (10A), A1 (1999).

[22] V. Krakoviack, Phys. Rev. Lett. 94 (6), 065703 (2005).

[23] V. Krakoviack, Phys. Rev. E 75 (3), 031503 (2007).

[24] V. Krakoviack, Phys. Rev. E 79 (6), 061501 (2009).

[25] J. Kurzidim, D. Coslovich and G. Kahl, Phys. Rev. Lett. 103 (13), 138303 (2009).

[26] K. Kim, K. Miyazaki and S. Saito, EPL 88 (3), 36002 (2009).

[27] J. Kurzidim, D. Coslovich and G. Kahl, Phys. Rev. E 82 (4), 041505 (2010).

[28] J. Kurzidim, D. Coslovich and G. Kahl, arXiv:1012.1267 (2010).

[29] A.R. Kerstein, J. Phys. A: Math. Gen. 16, 3071 (1983).

[30]S. Sastry, D.S. Corti, P.G. Debenedetti and F.H. Stillinger, Phys. Rev. E 56 (5), 5524 (1997).

[31]B.J. Sung and A. Yethiraj, J. Chem. Phys. 128 (5), 054702 (2008).

[32] S. Babu, J.C. Gimel and T. Nicolai, J. Phys. Chem. B 112 (3), 743 (2008).

[33] B.J. Alder and T.E. Wainwright, J. Chem. Phys. 31 (2), 459 (1959).

[34]D.C. Rapaport, The Art of Molecular Dynamics Simulation, 2nd ed. (Cambridge University Press, Cambridge, 2004).

[35]F.P. Preparata and M.I. Shamos, Computational geometry: an introduction (Springer-Verlag, New York, 1985).

[36]D. Stauffer and A. Aharony, Perkolationstheorie: eine Einführung (VCH, Weinheim, 1995). 
[37] J. Mittal, J.R. Errington and T.M. Truskett, J. Phys. Chem. B 110 (37), 18147 (2006).

[38] L.L. Lee, J. Chem. Phys. 103 (21), 9388 (1995).

[39] N.F. Carnahan and K.E. Starling, J. Chem. Phys. 51 (2), 635 (1969).

[40]D.P. Landau and K. Binder, A Guide to Monte Carlo Simulations in Statistical Physics (Cambridge University Press, Cambridge, 2000).

[41]R. Chang, K. Jagannathan and A. Yethiraj, Phys. Rev. E 69 (5), 051101 (2004).

[42] K. Kim, K. Miyazaki and S. Saito, Eur. Phys. J. Spec. Top. 189 (1), 135 (2010).

[43] S. Sastry, P.G. Debenedetti and F.H. Stillinger, Phys. Rev. E 56 (5), 5533 (1997). 